Engineering Physics and Mathematics Division

Mathematical Sciences Section

\title{
A LOOK AT SCALABLE DENSE LINEAR ALGEBRA LIBRARIES
}

Jack J. Dongarra $\$$ \$

Robert van de Geijn ${ }^{4}$

David W. Walker \$

$\$$ Department of Computer Science 107 Ayres Hall Knowville, TN 37996-1301

† Department of Computer Sciences University of 'Texas Austin, TX 78712

$\S$ Mathematical Sciences Sectio: Oak Ridge National Laboratory P.O. Box 2008, Bldg. 6012 Oak Ridge, TN 37831-6367

Date Published: July 1992

Research was supported by the Applied Mathematical Sciences Research Program of the Office of Energy Research, U.S. Department of Energy, and by the Defense Advanced Research Projects Agency under contract DAAL03-91-C-0047.

Prepared by the

Oak Ridge National Laboratory

Oak Ridge, Tennessee 378.31

managed by

Martin Marietta Energy Systems, Ine.

for the

US. DEPARTMENT OF ENERGYY

under contrace No. DE-AC05-84OR21400 


\section{Contents}

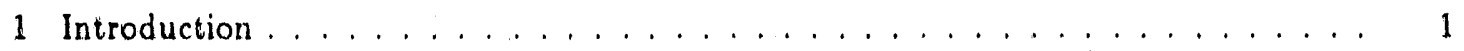

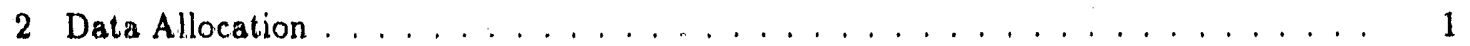

3 An Example . . . . . . . . . . . . . . . . . . . . . . 7

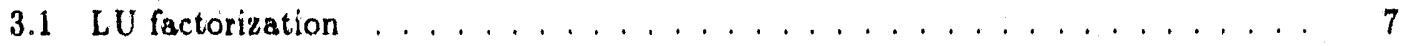

3.2 Experiments on the Intel Delta $\ldots \ldots \ldots \ldots \ldots$

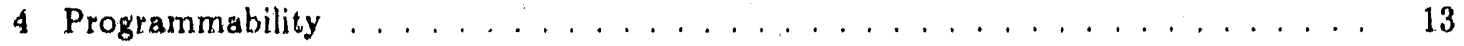

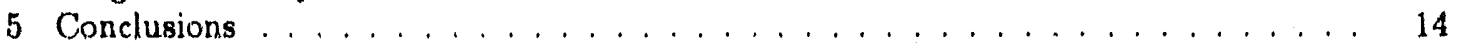

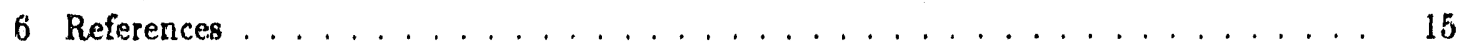




\title{
A LOOK AT SCALABLE DENSE LINEAR ALGEBRA LIBRARIES
}

\author{
Jack J. Dongarra \\ Robert van de Geijn \\ David W. Walker
}

\begin{abstract}
We discuss the essential design features of a library of scalable software for performing dense linear algebra computations on distributed memory concurrent computers. The square block scattered decomposition is proposed as a flexible and general-purpose way of decomposing most, if not all, dense matrix problems. An object-oriented interface to the library permits more portable applications to be written, and is easy to learn and use, since details of the parallel implementation are hidden from the user. Experiments on the Intel Touchstone Delta system with a prototype code that uses the square block scattered decomposition to perform LU factorization are presented and analyzed. It was found that the code was both scalable and efficient, performing at about $14 \mathrm{Gflop} / \mathrm{s}$ (double precision) for the largesi problem considered.
\end{abstract}




\section{Introduction}

Advanced parallelizing compilers may one day be capable of generating efficient parallel code for MIMD distributed memory concurrent computers (or multicomputers) from sequential code. However, in the interim, the development of scalable libraries is a key component in the development of a software environment that will allow the computational power of multicomputers to be exploited, and made available to a broader community of users. Over the next few years we envisage such libraries being developed in a number of areas, and that they will be accessible through a variety of interfaces. This paper focuses on issues impacting the design of scalable libraries for performing dense linear algebra on multicomputers. However, we believe that many of the issues discussed here are applicable to scalable libraries in other areas, and, indeed, it is important to impose some uriformity upon the design of different libraries.

In the next section we discuss data allocation, that is, how the data iterns in a parallel program are laid out in the hierarchical memory of the concurrent computer. 'The block scattered decomposition will be shown to encompass a large class of decompositions, and to provide sufficient flexibility for essentially all dense linear algebra computations. In Section 3 we use the right-looking variant of the $L U$ factorization algorithm for dense matrices to demonstrate the block scattered decomposition for a specific well-known example. A brief discussion of the run-time analysis of the algorithm is given, together with results of experiments running at up to $14 \mathrm{Gflop} / \mathrm{s}$ on the Intel Touchstone Delta system. Section 4 deals with programmability and implementation issues, and will discuss an objected-oriented approach to scalable libraries. Conclusions are presented in Section 5.

\section{Data Allocation}

The layout of an application's data within the hierarchical memory of a concurrent computer is critical in determining the performance and scalability of the parallel code. On shared memory concurrent computers (or multiprocessors) there are at least three levels to the memory hierarchy: the shared memory, and each processor's cache and registers. On such machines efficient codes seek to maximize the cache hit ratio, i.e., to avoid having to reload the cache too frequently. The software package LAPACK $[1,8]$ does this by casting linear algebra computations in terms of block-oriented, matrix-matrix operations known as the Level 3 BLAS $[10,11]$ whenever possible. This approach generally results in high cache hit ratios, without requiring any explicit cache manipulation by the application programmer. One of the aims of our work is to investigate a distributed memory version of LAPACK

There are also levels to the memory hierarchy on multicomputers: the local and nonlocal (remote) memory. In addition, each processor may have a hierarchical memory. Each processor has its own local memory, and the nonlocal memory for a given processor is simply the local 
memory of the other processors. A processor plus its local memory and other closely coupled hardware is refered to as a node. The nodes of a multicomputer are connected via a communication network; there is no physical shared memory. There are two important differences between multiprocessors and multicomputers. The first is that multiprocessors are generally faster than multicomputers in transfering data between two layers of the memory hierarchy. In particular, MIMD multicomputers typically incur a high communication latency. The second difference is that while bus-based multiprocessors usually have no more than 20 or 30 processors, multicomputers typically have several hundred to a few thousand processors. Thus the processors of a multiprocessor are large grain size and closely coupled, whereas those of a multicomputer are of smaller grain size and are less closely coupled. This means that the programming techniques and algorithms that are succassful on multiprocessors may not result in scalable codes on multicomputers.

On a multicomputer the application programmer is responsible for distributing (or decompasing) the data over the nodes of the concurrent computer. A vector of length $M$ may be decomposed over some set of $N_{p}$ nodes by first arranging the nodes in a linear sequence, and then assigning the vector entry with global index $m$ (where $0 \leq m<M$ ) to the pth node in the sequence $\left(0 \leq p<N_{p}\right)$, where it is stored as the ith entry in a local array. Thus the decomposition of a vector can be regarded as a mapping of the global index, $m$, to an index pair, $(p, i)$, specifying the node location and the local index.

For matrix problerns one can think of arranging the nodes as a $P$ by $Q$ grid. Thus the grid consists of $P$ rows of nodes and $Q$ columns of nodes, and $N_{p}=P Q$. Each node can be uniquely identified by its position, $(p, q)$, on the node grid. The decomposition of an $M \times N$ matrix can be regarded as the tensor product of two vector decompositions, $\mu$ and $\nu$. 'The mapping $\mu$ decomposes the $M$ rows of the matrix over the $P$ rows of nodes, and $\nu$ decomposes the $N$ columns of the matrix over the $Q$ columns of nodes. Thus, if $\mu(m)=(p, i)$ and $\nu(n)=(q, j)$ then the matrix entry with global index $(m, n)$ is assigned to the node at position $(p, q)$ on the node grid, where it is stored in a local array with index $(i, j)$.

Two common decompositions are the block and the scattered decompositions $[7,18]$. The block decomposition, $\lambda$, assigns contiguous entries in the global vector to the nodes in blocks.

$$
\lambda(m)=(\lfloor m / L\rfloor, m \bmod L),
$$

where $L=\lceil M / P\rceil$. The scattered decomposition, $\sigma$, assigns consecutive entries in the global vector to different nodes,

$$
\sigma(m)=(m \bmod P,\lfloor m / P\rfloor)
$$

Figures 1 and 2 show exanples of a $10 \times 10$ matrix decomposed over one and 1 wo-dimensional 
processor meshes. Various combinations of block and scattered decompositions are shown.

Two features that are desirable in a parallel subroutine library are;

1. a large degree of decomposition independence, so that a subroutine will work correctly for a large class of decompositions of the input data,

2. a set of communication routines for transforming between different decompositions.

These components give the application programmer the option of changing the decomposition, if necessary, so that a given phase of the computation can be performed optimally, i.e., with the least concurrent overhead. Alternatively, the programmer may choose to leave the decomposition unchanged and perform the computation suboptimally, thereby avoiding the overhead associated with changing the decomposition. The important point here is that the software should be sufficiently flexible to permit the programmer to make the choice, rather than irnposing a particular method.

Decomposition-independence could be achieved by having the subroutine contain a conditional statement, with each clause corresponding to a different type of decomposition. A more elegant and, we believe, better approach is to use a block scattered decomposition that is able to reproduce all the decompositions in Figs. 1 and 2, except for those shown in Figs. 2(b) and (c). In the block scattered approach blocks of $r$ elements are scattered over the nodes instead of single elements. The mapping of the global index, $m$, can be expressed as a triplet of values, $\mu(m)=(p, t, i)$, where $p$ is the node position, $t$ the block number, and $i$ the local index within the block. For the block scattered decomposition we may write,

$$
\zeta_{r}(m)=\left(\left\lfloor\frac{m \bmod T}{r}\right\rfloor,\left\lfloor\frac{m}{T}\right\rfloor,(\operatorname{mog} \bmod T) \bmod r\right)
$$

where $T=r P$. It should be noted that this reverts to the scattered decomposition when $r=1$, with local block index $i=0$. A block decomposition is recovered when $r=L$, with block number $t=0$. The block scattered decomposition in one form or another has previously been used by Saad and Schultz [20], Skjellum and Leung [21], Dongarra and Ostrouchov [9], Anderson et al. [2], Ashcraft [4,5], Dongarra and van de Geijn [15], van de Geijn [22], and Brent [6], to name a few. The block scattered decomposition is one of the decompositions provided in the Fortran D programming style [17].

As discussed above, the block scattered decomposition of a matrix can be regarded as the tensor product of two block scattered decompositions, $\mu_{r}$ and $\nu_{\alpha}$. This results in scaltered blocks of size $r \times s$. We can view the block scattered decomposition as stamping a $P \times Q$ processor grid, or template, over the matrix, where each cell of the grid covers $r \times s$ data items, and is labeled by its position in the template. In Table 1 we give the values of the block size $r \times s$ that give the same results as the block and scattered decompositions in Figs. 1 and 2 . 


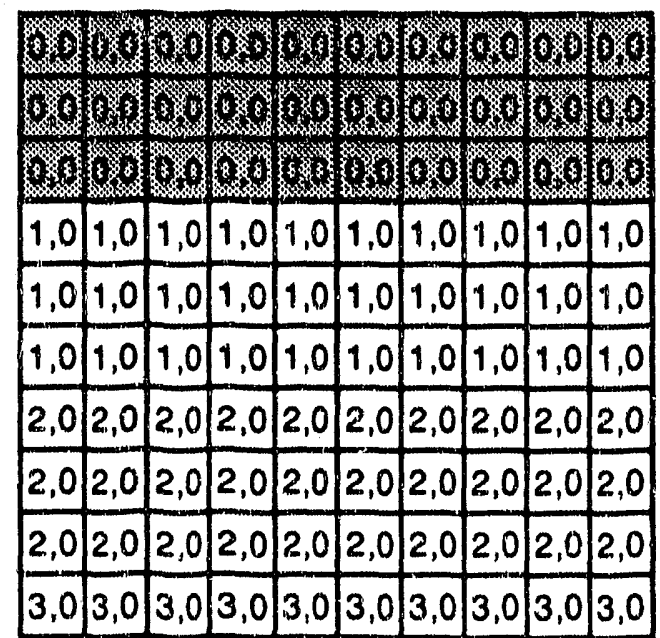

(a) $\mu$ block, $P=4, Q=1$

\begin{tabular}{|c|c|c|c|c|c|c|c|c|c|}
\hline 9 & 4 & 6 & 0,1 & 0,1 & 0,1 & 0,2 & 0,2 & 0,2 & 0,3 \\
\hline 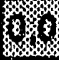 & 6 & (6) & 0,1 & $|0,1|$ & $0,1\}$ & 0,2 & 0,2 & $\mid 0,2$ & 0,3 \\
\hline 20 & 68 & $\%$ & 0,1 & 0,1 & 0,1 & 0,2 & $\mid 0,2$ & 0,2 & 0,3 \\
\hline 83 & $1 / 3$ & 6 & 0,1 & $(0,1)$ & 0,1 & 0,2 & $|0,2|$ & $|0,2|$ & 0,3 \\
\hline 80 & 160 & 64 & 0,1 & 0,1 & 0,1 & 0,2 & 0,2 & $0,2 \mid$ & 0,3 \\
\hline 8 & 16 & 688 & 0,1 & 0,1 & 0,1 & $\mid 0,2$ & 0,2 & $\mid 0,2$ & 0,3 \\
\hline 40 & 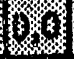 & 68 & 0,1 & $(0,1)$ & $|0,1|$ & 0,2 & $0,2\}$ & {$[0,2]$} & 0,3 \\
\hline 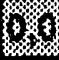 & W & 6,0 & 0,1 & $(0,1)$ & $\mid 0,1]$ & 0,2 & $\mid 0,2\}$ & 0,2 & 0,3 \\
\hline (8) & (6) & 180 & 0,1 & 0,1 & 0,1 & 0,2 & 0,2 & 0,2 & 0,3 \\
\hline 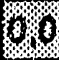 & 69 & 100 & 0,1 & 0,1 & $(0,1)$ & 0,2 & $(0,2)$ & $(0,2)$ & 0,3 \\
\hline
\end{tabular}

(c) $\vee$ block, $P=1, Q=4$

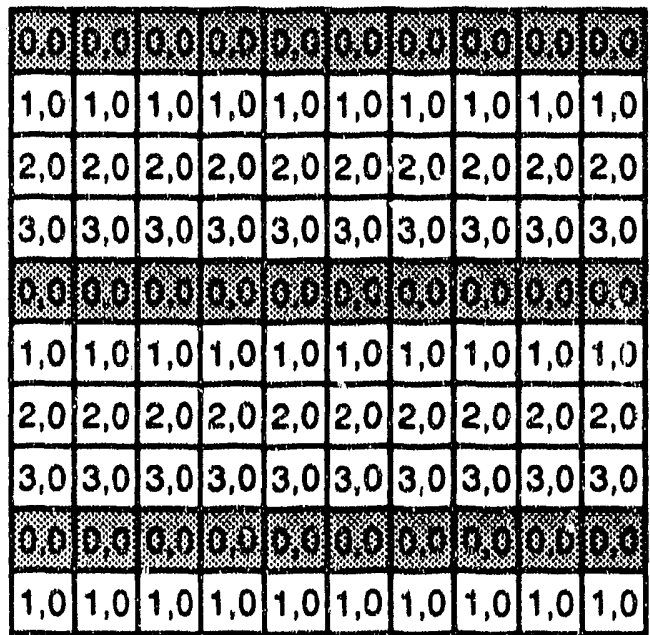

(b) $\mu$ scattered, $P=4, Q=1$

\begin{tabular}{|c|c|c|c|c|c|c|c|c|c|}
\hline 9 & 0,1 & 0,2 & 0,3 & 4 & 0,1 & 0,2 & 0,3 & \% & 0,1 \\
\hline 8 & 0,1 & 0,2 & 0,3 & 4 & 0,1 & 0,2 & 0,3 & 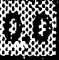 & \\
\hline 1 & 0,1 & 0,2 & 0,3 & 4 & 0,1 & 0,2 & $\mid 0,3)$ & 61 & 0,1 \\
\hline 0 & 0,1 & $|0,2|$ & 0,3 & \% & 0,1 & {$[0,2$} & {$[0,3\}$} & 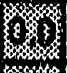 & \\
\hline 20 & $(0,1)$ & $\mid 0,2$ & 0,3 & (6) & 0,1 & 0,2 & 0,3 & \% & 0,1 \\
\hline 80 & 0,1 & $\mid 0,2$ & 0,3 & 40 & 0,1 & $(0,2)$ & 0,3 & 96 & 0,1 \\
\hline 3 & 0,1 & 0,2 & 0,3 & (x) & 0,1 & {$[0,2]$} & $0,3)$ & 6 & 0,1 \\
\hline 21 & 0,1 & 0,2 & $(0,3)$ & 8,4 & 0,1 & 0,2 & {$[0,3)$} & . & 0,1 \\
\hline 89 & 0,1 & 0,2 & 0,3 & 6 & 0,1 & 0,2 & $(0,3)$ & 8,1 & 0,1 \\
\hline & $(0,1)$ & $(0,2)$ & & 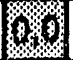 & {$[0,1]$} & $|0,2|$ & $|0,3|$ & 100 & \\
\hline
\end{tabular}

(d) v scattered, $P=1, Q=4$

Figure 1: These 4 figures show different ways of decomposing a $10 \times 10$ matrix over a onedimensional processor mesh. Each cell represents a matrix entry, and is labeled by the position, $(p, q)$, in the node grid of the node to which it is assigned. To emphasize the pattern of decomposition the matrix entries assigned to the node in the first row and column of the node grid are shown shaded. Figures (a) and (b) show block and scattered row-oriented decompositions, respectively, for 4 nodes arranged as a $4 \times 1$ grid $(P=4, Q=1)$. In figures (c) and (d) the corresponding column-oriented decompositions are shown $(P=1, Q=4)$. 


\begin{tabular}{|c|c|c|c|c|c|c|c|c|c|}
\hline , & k & (K) & 0,1 & $\mid 0,1]$ & 0,1 & 0,2 & $0,2)$ & $\mid 0,2]$ & 0,3 \\
\hline 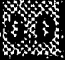 & SON & 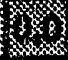 & 0,1 & $0,1 \mid$ & 0,1 & {$[0,2)$} & 0,2 & 0,2 & 0,3 \\
\hline six & 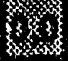 & 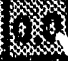 & $(0,1$ & 0,1 & 0,1 & 0,2 & $|0,2|$ & 0,2 & 0,3 \\
\hline 1,0 & $1,0\}$ & 1,0 & 1,1 & 1,1 & 1,1 & 1,2 & $|1,2|$ & 1,2 & 1,3 \\
\hline 1,0 & $\mid 1,0$ & 1,0 & $\mid 1,1$ & 1,1 & 1,1 & 1,2 & $|1,2|$ & 1,2 & 1,3 \\
\hline 1,0 & 1,0 & 1,0 & $\mid 1,1$ & 1,1 & 1,1 & 1,2 & $|1,2|$ & $\mid 1,2$ & 1,3 \\
\hline 2,0 & 2,0 & 2,0 & 2,1 & 2,1 & 2,1 & 2,2 & $|2,2|$ & 2,2 & 2,3 \\
\hline 2,0 & 2,0 & 2,0 & 2,1 & 2,1 & 2,1 & 2,2 & $|2,2|$ & 2,2 & 2,3 \\
\hline 2,0 & 2,0 & 2,0 & 2,1 & 2,1 & $2,1\}$ & 2,2 & $2,2]$ & 2,2 & 2,3 \\
\hline 3,0 & 3,0 & 3,0 & 3,1 & 3,1 & 3,1 & 3,2 & 3,2 & 3,2 & 3,3 \\
\hline
\end{tabular}

(a) $\mu$ block, $v$ block, $P=Q=4$

\begin{tabular}{|l|l|l|l|l|l|l|l|l|l|}
\hline & & & 0,1 & 0,1 & 0,1 & 0,2 & 0,2 & 0,2 & 0,3 \\
\hline 1,0 & 1,0 & 1,0 & 1,1 & 1,1 & 1,1 & 1,2 & 1,2 & 1,2 & 1,3 \\
\hline 2,0 & 2,0 & 2,0 & 2,1 & 2,1 & 2,1 & 2,2 & 2,2 & 2,2 & 2,3 \\
\hline 3,0 & 3,0 & 3,0 & 3,1 & 3,1 & 3,1 & 3,2 & 3,2 & 3,2 & 3,3 \\
\hline & & & 0,1 & 0,1 & 0,1 & 0,2 & 0,2 & 0,2 & 0,3 \\
\hline 1,0 & 1,0 & 1,0 & 1,1 & 1,1 & 1,1 & 1,2 & 1,2 & 1,2 & 1,3 \\
\hline 2,0 & 2,0 & 2,0 & 2,1 & 2,1 & 2,1 & 2,2 & 2,2 & 2,2 & 2,3 \\
\hline 3,0 & 3,0 & 3,0 & 3,1 & 3,1 & 3,1 & 3,2 & 3,2 & 3,2 & 3,3 \\
\hline & 3,0 & 0 & 0,1 & 0,1 & 0,1 & 0,2 & 0,2 & 0,2 & 0,3 \\
\hline 1,0 & 1,0 & 1,0 & 1,1 & 1,1 & 1,1 & 1,2 & 1,2 & 1,2 & 1,3 \\
\hline
\end{tabular}

(c) $\mu$ scattered, $\vee$ block, $P=Q=4$

\begin{tabular}{|c|c|c|c|c|c|c|c|c|c|}
\hline 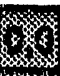 & 0,1 & $\mid 0,2$ & 0,3 & $\Leftrightarrow 8$ & 0,1 & 0,2 & 0,3 & 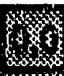 & \\
\hline $8 z$ & 0,1 & 0,2 & 3 & 68 & 0,1 & 0,2 & 3 & $k$ s & \\
\hline ל & 0,1 & $\mid 0,2)$ & & (3) & 0,1 & 0,2 & 0,3 & 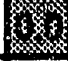 & \\
\hline & 1,1 & $|1,2|$ & 3 & 1,0 & 1,1 & 1,2 & 1,3 & 1,0 & \\
\hline 0 & $\mid 1,1$ & 1,2 & 1,3 & 1,0 & 1,1 & 1,2 & 1,3 & 110 & \\
\hline & 1,1 & $|1,2|$ & 3 & $|1,0|$ & 1,1 & 1,2 & 1,3 & & \\
\hline & 2,1 & $|2,2|$ & 3 & 2,0 & 2,1 & 2,2 & 2,3 & 2,0 & \\
\hline & 2,1 & 2,2 & 2,3 & 2,0 & 2,1 & 2,2 & 2,3 & 2,0 & \\
\hline & lo & 2,2 & 2,3 & 2,0 & 2,1 & 2,2 & 2,3 & 10,0 & \\
\hline & & & & & & & & & \\
\hline
\end{tabular}

(b) $\mu$ block, $v$ scattered, $P=Q=4$

\begin{tabular}{|c|c|c|c|c|c|c|c|c|c|}
\hline 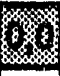 & {$[0,1]$} & $|0,2|$ & 0,3 & 63 & 0,1 & $\mid 0,2$ & 0,3 & 5 & \\
\hline & $|1,1|$ & $|1,2|$ & $|1,3|$ & $|1,0|$ & 1,1 & 1,2 & 1,3 & 1,0 & \\
\hline & 1 & 2,2 & 3 & .0 & 1 & 2 &, 3 & 80 & \\
\hline & 3,1 & 3,2 & 3,3 & 3,0 & 3,1 & 3,2 & 3,3 & 3,0 & \\
\hline & 0,1 & $|0,2|$ & $|0,3|$ & $6 \times 4$ & 0,1 & 0,2 & 0,3 & 6 & \\
\hline & 1,1 & 1,2 & 1,3 & 1,0 & 1,1 & 1,2 & 1,3 & 140 & \\
\hline & $2,1 \mid$ & 2,2 & $2,3 \mid$ & 2,0 & 2,1 & 2,2 & 2,3 & $J<, 0$ & \\
\hline & 3,1 & 3,2 & 3,3 & $|3,0|$ & 3,1 & 3,2 & 3,3 & 3,0 & \\
\hline & 0,1 & 0,2 & 0,3 & 62 & In 1 & 0,2 & 0,3 & 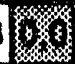 & \\
\hline & & & & & & & & & \\
\hline
\end{tabular}

(d) $\mu$ scattered, $v$ scattered, $P=Q=4$

Figure 2: These 4 figures show different ways of decomposing a $10 \times 10$ matrix over a t,wodimensional processor mesh of 16 nodes arranged as a $4 \times 4$ grid $(P=Q=4)$. 


\begin{tabular}{|c|c|c|c|c|c|}
\hline Figure & $P$ & $Q$ & $r$ & $s$ & $r=s$ \\
\hline $1(\mathrm{a})$ & 4 & 1 & 3 & 10 & 3 \\
$1(\mathrm{~b})$ & 4 & 1 & 1 & 0 & 1 \\
$1(\mathrm{c})$ & 1 & 4 & 10 & 3 & 3 \\
$1(\mathrm{~d})$ & 1 & 4 & 10 & 1 & 1 \\
$2(\mathrm{a})$ & 4 & 4 & 3 & 3 & 3 \\
$2(\mathrm{~b})$ & 4 & 4 & 3 & 1 & - \\
$2(\mathrm{c})$ & 4 & 4 & 1 & 3 & - \\
$2(\mathrm{~d})$ & 4 & 4 & 1 & 1 & 1 \\
\hline
\end{tabular}

Table 1: Block-scat tered decomposition parameters needed to reproduce the block and scattered decompositions in ligs. 1 and 2. The last column gives the block size when only square blocks are used. Decompositions 2(b) and 2(c) cannot be generated with square blocks.

The block and scattered decompositions may be regarded as special cases of the block scattered decomposition. In general, the scattered blocks are rectangular, however, the use of nonsquare blocks can lead to complications. For example, in the LU factorization algorithm, described in the next section, a triangular solve is needed to update submatrix $C$. If nonsquare blocks are used either the triangular matrix will extend over more than one column of blocks (if $r>s$ ), or the submatrix $C$ will extend over more than one row of blocks (if $r<s$ ). Thus, nonsquare blocks will result in additional software and communication overhead. We, therefore, propose to restrict ourselves to the square block scattered (SBS) class of decompositions. The column and row decompositions can still be recovered by setting $P=1$ or $Q=1$, as shown in Table 1, however, the decompositions shown in Figs. 2(b) and (c) cannot be generated with an SBS decomposition.

So far we have only considered how to map matrix elements onto the node grid. In decomposing a problem we must also specify how locations in the node grid are mapped to physical nodes. Common mapping functions are the natural mapping,

$$
A(i, j)=i+j \cdot Q
$$

and the binary-reflected Gray code mapping,

$$
A(i, j)=G(i)+G(j) \cdot Q
$$

where $G(x)$ denotes the Gray code of $x$, and $i=0,1, \ldots, Q-1, j=0,1, \ldots, P-1$. On most current multicomputers the cost of communicating between any two nodes is weakly dependent of their separation in the topology of the comnunication network. Hence the choice of mapping should not impact performance very much. The subroutine library should support the natural 
and Gray code mappings, as well as any function, $A$, supplied by the application programmer.

\section{An Example}

In this section, we discuss the scalability of the $\mathrm{LU}$ factorization algorithm when it is implemented using the block scattered decomposition. First, we describe the algorithm. Next, we summarize the results from an analysis of the time complexity. Data from experiments on the Intel Touchstone Delta system are used to further demonstrate the scalability.

\subsection{LU factorization}

To obtain our parallel implementation of the LU factorization, we started with a variant of the right-looking LAPACK LU factorization routine. It can be briefly described as follows: Assume the $L U$ factorization has proceeded so that all but the labeled portions of the matrix have been updated:

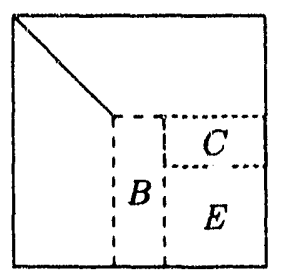

where $B \in \mathbf{R}^{M \times r}, C \in \mathbf{R}^{r \times(M-r)}$, and $E \in \mathbf{R}^{(M-r) \times(M-r)}$. During the next step, the rightlooking algorithm factors panel $B$, pivoting if necessary. Next, the pivots are applied to the remainder of the matrix. Blocks $C$ and $E$ now become blocks $\bar{C}$ and $\bar{E}$, a triangular solve updates submatrix $\bar{C}$, and a rank $r$ update updates submatrix $\bar{E}$. This process continues recursively with the updated matrix [12].

Turning now to the distributed memory implementation, assume the matrix is distributed among a $P \times Q$ grid of nodes using a block scattered decomposition, with block size $r \times r$. For our analysis, we assume that communicating a block of $k$ floating point numbers between any two nodes requires time $\alpha+k \beta$, where $\alpha$ and $\beta$ represent the communication latency and the inverse of the bandwidth, respectively. In addition, the time for a floating point operation is given by $\gamma$.

The above descrited process proceeds as follows:

- ( $\mathrm{f} B$ ) The column of nodes that holds $B$ collaborates to factor this panel. Since there is relatively little to compute (the panel is typically narrow), and communication is restricted to short messages, the contribution of this operation to the run-time is almost entirely due to communication latency. We will ignore the other costs. For each column, this consists of $\log (P) \alpha$ for determining the pivot row, $\alpha$ for swapping pivot rows of this panel, and 
another $\log (P) \alpha$ for broadcasting the pivot row. (Possible optimization: since this is latency bound, a clever implementation would combine the messages for determining the pivot row, and distributing it within the column of nodes that hold the panel.)

- (bp) Pivot information is distributed to all other columns of nodes. Approximate contribution to run-time: $\alpha$ per panel.

- (p) Columns of nodes collaborate to pivot the remainders of the matrix rows. Approximate contribution to run-time:

$$
r(\alpha+\lceil(N-r) / Q\rceil \beta)
$$

for panel $k=1, \ldots, N / r$.

- $(b \bar{B})$ Factored panel $B$ is distributed within rows of nodes. Approximate contribution to run-time:

$$
2(\alpha+[(N-(k-1) r) / P\rceil r \beta)
$$

for panel $k=1, \ldots, N / r$. (Since this operation can be pipelined around the ring, overlapping with computation, there is no $\log (Q)$ term here.)

- $(\mathrm{b} \bar{C})$ The row that holds $\bar{C}$ performs the triangular solve, the results of which are distributed within columns of nodes. Approximate contribution:

$$
\lceil(N-k r) / Q\rceil r^{2} \gamma+\log (P)(\alpha+\lceil(N-k r) / Q\rceil r \beta)
$$

for panel $k=1, \ldots, N / r$.

- $(u \bar{E})$ Most parallelism is derived from updating $\bar{E}$. Approximate contribution:

$$
2\lceil(N-k r) / P\rceil\lceil(N-k r) / Q\rceil r \gamma
$$

for panel $k=1, \ldots, N / r$.

The total run time is then given by

$$
T_{\mathrm{tot}} \approx T_{\mathrm{fB}}+T_{\mathrm{tp}}+T_{\mathrm{p}}+T_{\mathrm{bB}}+T_{\mathrm{fC}}+T_{\mathrm{uE}}
$$

where the different terms come from summing over all panels the different contributions given above.

Since the total computation time of the algorithm on a single processor is given by $T_{1} \approx$ $(2 / 3) N^{3} \gamma$, the efficiency attained, $E=T_{1} / p T_{t o t}$, as a function of the various parameters, can 
be shown to be of the form

$$
E=\left[1+\frac{p}{N^{2}}\left(c_{1} \log (P)+c_{2}\right) \frac{\alpha}{\gamma}+\frac{P}{N}\left(c_{3} \log (P) \frac{\beta}{\gamma}+c_{4}\right)+\frac{Q}{N}\left(c_{5} \frac{\beta}{\gamma}+c_{6}\right)\right]^{-1}
$$

where $c_{1-6}$ depend only on $r$.

Let us start by considering the block column scattered decomposition, i.e., $P \times Q=1 \times p$. Then, for reasonably large $N$,

$$
E \approx\left[1+c_{2} \frac{p}{N^{2}} \frac{\alpha}{\gamma}+\frac{p}{N}\left(c_{5} \frac{\beta}{\gamma}+c_{6}\right)\right]^{-1}
$$

In the limit, $N$ must grow with $p$ to maintain efficiency. Notice that the $N^{2}$ cannot be readily ignored, even for $N=O\left(10^{3}\right)$, since $\alpha$ is several orders of magnitude greater than $\gamma$ for many multicomputers. This kind of scalability poses a problem: Memory requirements grow with $N^{2}$ and hence eventually $N$ cannot be increased to maintain efficiency. A similar analysis can be done for row distributions.

By contrast, consider a general $P \times Q$ grid of nodes. Assume the ratio $Q / P$ is kept constant as $p$ is increased, i.e., $P=u \sqrt{p}$ and $Q=v \sqrt{p}$, where $u$ and $v$ are constants. Then $P / N$ and $Q / N$ become $u \sqrt{p} / N$ and $v \sqrt{p} / N$, respectively. If $\log (P)$ is ignored, since it is a slowly growing function, $N^{2}$ must grow with $p$ in order to maintain efficiency. If $\log (P)$ is not ignored, it can be argued that once $P$ is sufficiently large (e.g., greater than 4) performance will degrade slowly with $p$.

\subsection{Experiments on the Intel Delta}

In this section, we discuss results from experiments conducted on the Intel Touchstone Delta that illustrate the scalability of the LU factorization.

The Intel Touchstone Delta system is a distributed-memory, message-passing multicomputer of the Multiple Instruction Multiple Data (MIMD) class [19]. It consists of 520 i860-based nodes, interconnected via a communications network having the topology of a two-dimensional rectangular grid. The interconnection network employs a Mesh Routing Chip (MRC) at each system node. The peak interprocessor communications bandwidth is $\approx 30 \mathrm{MBytes} / \mathrm{s}$ in each direction. The system supports explicit message-passing, with a latency of $\approx 75$ microseconds via wormhole routing using a packet-based protocol. Interconnect blocking is minimized by interleaving packets associated with distinct messages that need to traverse the same interconnect path.

There are a number of issues that complicate a direct comparison of our analytical estimates and observed performance. First, certain optimizations can be done to improve the algorithm given in Section 3 [22], details of which go beyond the scope of this paper. Second, the parameter $\gamma$ is affected by the size of the data being manipulated: computation at different stages involves 


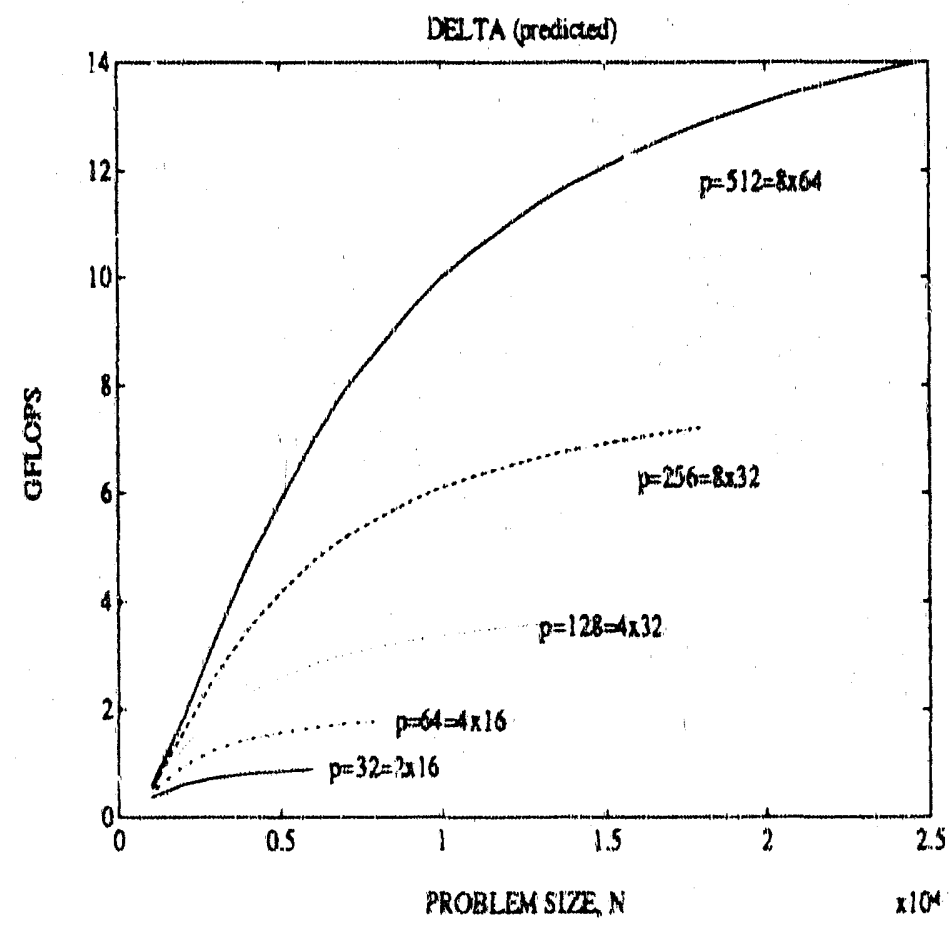

Figure 3: Total predicted performance for various $p$ as a function of the problem size $N$.

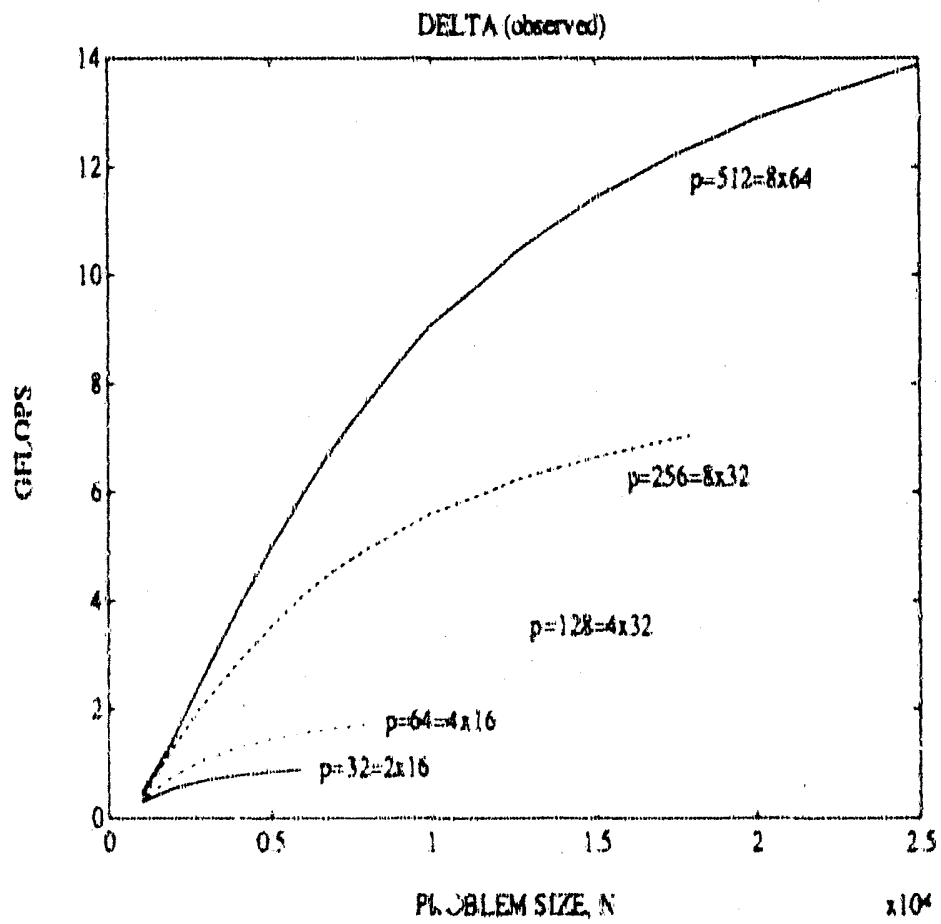

Figure 4: Total obsesved performani for various $p$ as a function of the problem size $N$. 


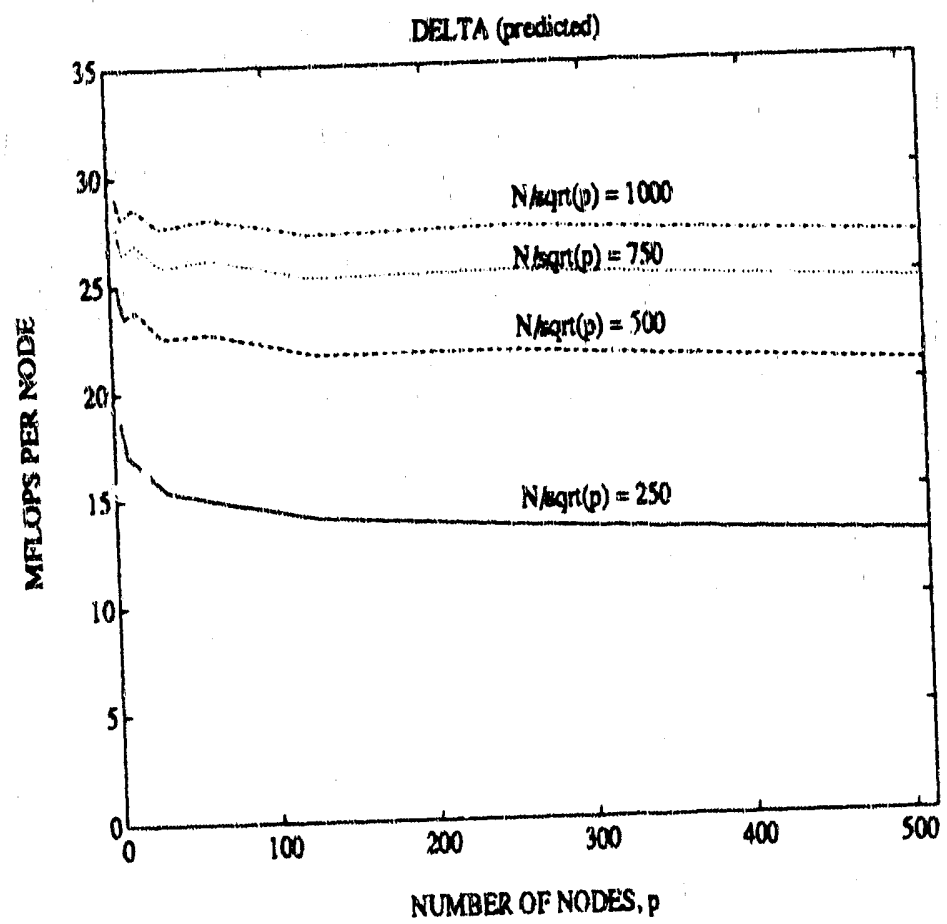

Figure 5: Predicted performance per node as the number of nodes $p$ varies. Different curves correspond to problem sizes increased so that $N^{2} / p$ is constant.

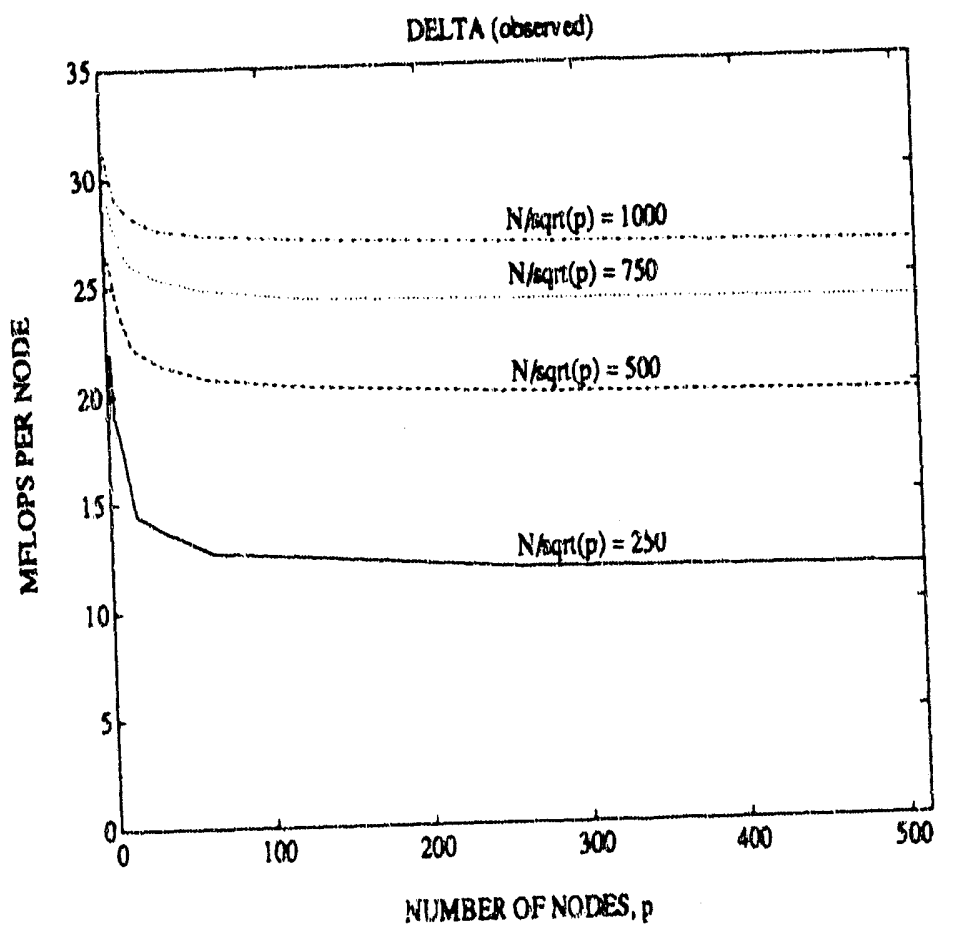

Figure 6: Performance per node attained as the number of nodes $p$ varies. 


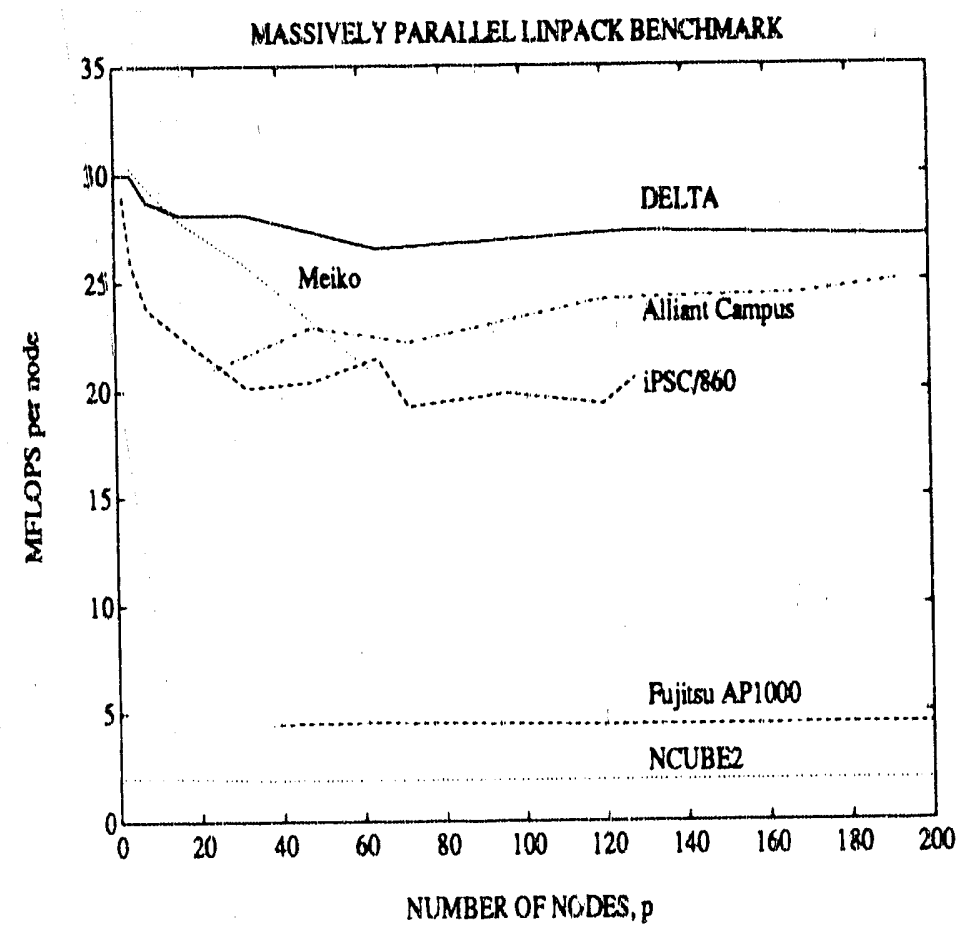

Figure 7: Performance per node attained for the LINPACK benchmark by various parallel architectures as the number of nodes $p$ varies.

Level 1,2, and 3 BLAS, which yield different performance depending on the size of the data being manipulated. Finally, the blocksize $r$ and grid size $P \times Q$ are chosen so the performance of the BLAS is maximized without creating unreasonable idle time due to load inbalance. This leaves us to investigate if the predicted trends can be observed in practice.

In Figs. 3-4, we report the predicted and observed performance of the LU factorization for different numbers of nodes when the problem size $N$ is varied. For the predicted performance, $\alpha=100 \mu \mathrm{sec}, \beta=1 \mu \mathrm{sec}$ ( $8 \mathrm{Mbytes} / \mathrm{sec}$ bandwidth), and $\gamma=29 \mathrm{nsec}$ (34 MFLOPS per node) where used. (These correspond roughly to what we observed in practice. Communication overhead is somewhat increased by our code.) The grid sizes were experimentally determined to be optimal for large problem sizes. As the problem size increases, performance improves. The results compare favorably with the peak performance that can be attained for this type of problem on the Delta.

The predicted degradation of performance when $N / \sqrt{p}$ is held constant is illustrated in Fig. 5. This trend is also observed in practice, as illustrated in Fig. 6. In these figures, we report efficiency as performance (in MFLOPS) per node.

The LU factorization is at the core of the LINPACK benchmark. This benchmark measures the performance of a given computer while performing a dense linear solve. A typical 
implementation 'starts by factoring the matrix, followed by triangular solves. Results from implementations on various parallel architectures are reported in [13]. To illustrate that the predicted trends can be observed on other parallel computers as well, we report performance per node in Fig. 7. While there is a clear incentive to fill the memory with the largest possible problem, thereby automatically increasing $N^{2}$ roughly with $p$, the data made available to us did not in all cases include problem sizes that scaled as nicely as those used for Fig. 6. Although data was available for an NCUBE2 up to size 1024, and for the Fujitsu and Delta up to size 512 , we concentrate on the more interesting range of machine sizes in this figure.

Several observations can be made: Both the NCUBE2 and the Fujitsu are based on relatively slow processors. This decreases the ratios $\alpha / \gamma$ and $\alpha / \beta$, thereby reducing the effects of communication overhead. Moreover, the performance of the BLAS on these machines is less affected by the size of the problem. All other machines are based on the same processor: the Intel i860. The curve for the Meiko follows the predicted trend, except that the last data point (for 62 nodes) is for a much smaller problem size than is required to keep $N^{2} / p$ constant. At first glance, the efficiency attained by the Alliant appears to improve with the number of nodes, defying the results of our analysis. Moreover, when looking at the raw data, the problem sizes actually grow slower than required by our analysis. This indicates that there is a lower order term that affects performance for small problem sizes. Indeed, it is reportedly due to an inefficient triangular solve algorithm used in this implementation.

\section{Programmability}

Programmability will be used here to refer to a number of features of the software environment concerned with software maintenance and usage. Programmability covers the flexibility, range of functionality, portablility, and ease of use of some software component. From an application programmer's point of view, the main factor that will determine how easy it is to learn and use the proposed subroutine library will be the interface to the subroutines. Clearly, this interface must pass the appropriate information about the decomposition and layout of the data in memory to the subroutine. This could be done in three ways:

1. by only allowing one type of decomposition for each subroutine so that different subroutines must be called for different decompositions. This avoids having to specify the decomposition in a lengthy argument list, but makes maintaining and porting the subroutine library rather tedious.

2. have a single subroutine handle all possible different decompositions and pass the decomposition information via the argument list. This can result in long argument lists. 
3. use an object-oriented approach in which a matrix is actually a data structure containing the data itself (or pointers to it), plus all the information necessary to fully specify the decomposition. This allows a single subroutine to handle all decompositions, and avoids a long argument list. This approach is the most elegant and conceptually simplest for the application programmer. It is rather more difficult to implement than the other two approaches.

The object-oriented approach allows details of the parallel implementation to be hidden at a low level of the software. Ideally, all communication would be hidden below the level of the BLAS routines. In the prototype parallel dense linear algebra library currently under development all interprocessor communication takes place explicitly at the level of the parallel linear algebra routines through calls to a communication library, the LACS routines $[3,16,14]$. Thus, currently the sequential BLAS routines, together with the LACS, are the building blocks used to build higher level library routines, such as $L U$ and $Q R$ factorization.

In addition to a vet of subroutines for performing matrix computations the proposed library will also contain routines for performing communication tasks. Such tasks will include global changes to the decomposition, such as performing a matrix transpose, and replicating parts of a matrix over group" of nodes. This latter type of communication is similar to the SPREAD routine in Fortran 90 [8], and will allow, for example, row and columns of a matrix to be communicated across the machine. These LACS could also be given an object-oriented style of interface. In fact, some of the array intrinsic functions of Fortran 90, such as SPREAD, CSHIFT, and EOSHIFT, could be included in the LACS.

Other utility routines will also be provided. One set of assignment routines will be used to initially specify the decomposition, and another set of inquiry routines will provide a means of extracting information about the current decornposition. These inquiry routines will allow application programmers to develop modular subprograms that are fully compatible with our linear algebra library.

\section{Conclusions}

The square block scattered decomposition (SBS) is a practical and general-purpose way of decomposing dense linear algebra computations. In problerns, such as LU factorization, in which rows and/or columns become inactive as the algorithm progresses, the SBS decomposition provides good load balance. At the same time it reduces communication latency since fewer messages need to be sent than in the nonblocked case $(r=1)$. It is possible to regard each of the blocks as a distinct process, so the SBS decomposition, in effect, overdecomposes the problem. The resultant parallel slackness could then be exploited by overlapping communication and computation. This might be a viable approach on future machines that support multithreading 
in the operating system kernel, or in hardware. However, on currently available machines the communication latency is probably too high to make it worthwhile, although our general approach should make it easy to exploit overdecomrosition in the future.

The LU factorization timinge presented in Section 3 show that the SBS decomposition results in scalable and efficient code, attaining a speed of about $14 \mathrm{Gflop} / \mathrm{s}$ on the Intel Touchstone Delta system for the largest problem considered.

We propose an object-oriented interface to the library routines, in which the objects are matrices that include pointers to both the matrix data and the decomposition. With this approach all interprocessor communication takes place within the Level 3 BLAS routines, or within the Linear Algebra Communication Subprograms (LACS), which are provided to perform common communication tasks. The user is largely insulated from the details of the parallel implementation, making applications more readily portable, and easier to develop.

\section{Acknowledgements}

This research was performed in part using the Intel Touchstone Delta System operated by the California Institute of Technology on behalf of the Concurrent Supercomputing Consortium. Access to this facility was provided by the California Institute of Technology and Intel Supercomputer Systems Divisinn.

\section{References}

[1] E. Anderson, Z. Bai, C. Bischof, J. Demmel, J. Dongarra, J. DuCroz, A. Greenbaum, S. Hammarling, A. McKenney, and D. Sorensen. Lapack: A portable linear algebra library for high-performance computers. In Proceedings of Supercompuling '90, pages 1-10. IEEE Press, 1990.

[2] E. Anderson, A. Benzoni, J. Dongarra, S. Moulton, S. Ostrouchov, B. Tourancheau, and

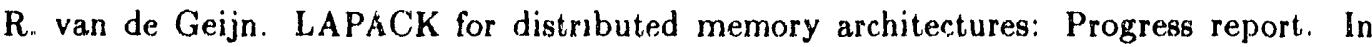
Parallel Processing for Scientific Computing, Fifth SIAM Conference. SIAM, 1991.

(3) E. Anderson, A. Benzoni, J. Dongarra, S. Moulton, S. Ostrouchov, B. Tourancheau, and R. van de Geijn. Basic Linear Algebra Communication Subprograms. In Sirth Distributed Memory Computing Conference Proceedings, pages 287--290. IEEE Computer Society Press, 1991

[4] C. C. Ashcraft. The distributed solution of linear systems using the torus wrap data mapping. Engineering Computing and Analysis Technical Report ECA-TR-147, Boeing Computer Services, 1990. 
[5] C. C. Ashcraft. A taxonamy of distributed dense LU factorization methods. Engineering Computing and Analysis Technical Report ECA-TR-161, Boeing Computer Services, 1991.

[6] R. Brent. The LINPACK' benchmark on the AP 1000: Preliminary repert. In Proceedings of the and CAP Workshop, NOV 1991.

[7] E. F. Van de Velde. Data redistribution and concurrency. Parallel Computing, 16, December 1990 .

[8] J. Demmel, J. J. Dongarra, J. Du Croz, A. Greenbaum, S. Hammarling, and D. Sorensen. Prospectus for the development of a linear algebra library for nigh performance computers. Technical Report 97, Argonne National Laboratory, Mathematics and Computır Science Division, September 1987.

[9] J. Dongarra and S. Ostrouchov. LAPACK block factorization algorithms on the Intel iPSC/860. Technical Report CS-90115, University of Tennessee at Knoxville, Computer Science Department, October 1990.

[10] J. J. Dongarra, J. Du Croz, S. Hammarling, and I. Duff. A set of level 3 basic linear algebra subprograms. ACM Transactions on Mathematical Software, 16(1):1-17, 1900.

[11] J. J. Dongarra, I. Duff, J. Du Croz, and S. Hammarling. A set of level 3 basic linear algebra subprograms. ACM TOMS, 16:1-17, March 1990.

[12] J. J. Dongarra, I. S. Duff, and D. C. Sorensen H. A. van der Vorst. Solving Linear Systems on Vector and Shared Memory Computers. SIAM, Philadelphia, PA, 1990.

[13] Jack J. Dongarra. Performance of various computers using standard linear equations software. Technical Report CS-89-85, University of Tennessee, October 1991.

[14] J.J. Dongarra. Workshop on the BIACS. LAPACK Working Note 34, Technical Report C.S-91-134, University of 'Tennessee, 1991

[15] J.J. Dongarra and R.A. van de Geijn. Reduction to condensed form for the eigenvalue problem on distributed memory architectures. LAPACK Working Note 30, Technical Report CS-91-130, University of Tennessec, 1991. To appear in Parallel Computing.

[16] J.J. Dongarra and R.A. van de Geijn. Two dimensional basic linear algebra communication subprograms. LAPACK Working Note 37, Technical Report CS-91-138, University of Tennessee, 1991.

[17] C. Fox, S. Hiranandani, K. Kennedy, C. Koelbel, U. Kremer, C-W. Tseng, and M-Y. Wu. Fortran D language specification. Technical Report CRPC-TR90079, Center for Research on Parallel Computation, Rice University, December 1990. 
[18] G. C. Fox, M. A. Johnson, G. A. Lyzenga, S. W. Otto, J. K. Salmon, and D. W. Walker. Solving Problems on Concurrent Processors, volume 1. Prentice Hall, Englewood Cliffs, N.J., 1988.

[19] S.L. Lillevik. The Touchstone 30 Gigaflop DELTA Prototype. In Sixth Distributed Memory Computing Conference Proceedings, pages 671-677. IEEE Computer Society Press, 1991.

[20] Y. Saad and M. H. Schultz. Parallel direct methods for solving banded linear systems. Technical Report YALEU/DCS/RR-387, Department of Computer Science, Yale University, 1985.

[21] A. Skjellum and A. Leung. LU factorization of sparse, unsymmetric, Jacobian matrices on multicomputers. In D. W. Walker and Q. F. Stout, editors, Proceedings of the Fifth Distributed Memory Concurrent Computing Comference, pages 328-337. IEEE Press, 1990.

[22] R.A. van de Geijn. Massively parallel LINPACK benchmark on the Intel Touchstone Delta and IPSC/860 systems. Computer Science report TR-91-28, Univ. of Texas, 1991. 


\section{INTERNAL DISTRIBUTION}

1. B. R. Appleton

2-3. T. S. Darland

4. E. F. D'Azevedo

5-9. J. J. Dongarra

10. G. A. Geist

11. L. J. Gray

12. M. R. Leuze

13. E. G. $\mathrm{Ng}$

14. C. E. Oliver

15. B. W. Peyton

16-20. S. A. Raby

21. C. H. Romine
22. T. H. Rowan

23-27. R. F. Sincovec

28-32. D. W. Walker

33-37. R. C. Ward

38. P. H. Worley

39. Central Research Library

40. ORNL Patent Office

41. K-25 Applied Technology Library

42. Y-12 Technical Library

43. Laboratory Records - RC

44-45. Laboratory Records Department

\section{EXTERNAL DISTRIBUTION}

46. Cleve Ashcraft, Boeing Computer Services, P.O. Box 24346, M/S 7L-21, Seattle, WA 98124-0346

47. Donald M. Austin, 6196 EECS Bldg., University of Minnesota, 200 Union Street, S.E., Minneapolis, MN 55455

48. Robert G. Babb, Oregon Graduate Institute, CSE Department, 19600 N.W. von Neumann Drive, Beaverton, OR 97006-1999

49. Lawrence J. Baker, Exxon Production Research Company, P.O. Box 2189, Houston, TX 77252-2189

50. Jesse L. Barlow, Department of Computer Science, Pennsylvania State University, University Park, PA 16802

51. Edward H. Barsis, Computer Science and Mathematics, P. O. Box 5800, Sandia National Laboratories, Albuquerque, NM 87185

52. Michael L. Barton, Intel Corporation, 15201 N. W. Greenbrier Parkway, Beaverton, OR 97006

53. Colin Bennett, Department of Mathematics, University of South Carolina, Columbia, SC 29208

54. Dominique Bennett, CERFACS, 42 Avenue Gustave Coriolis, 31057 Toulotie Cedex, FRANCE

55. Marsha J. Berger, Courant Institute of Mathematical Sciences, 251 Mercer Street, New York, NY 10012

56. Mike Berry, Department of Computer Science, University of 'Tennessee, 107 Ayres Hall, Knoxville, TN 37996-1301 
57. Chris Bischof, Mathematics and Cornputer Science Division, Argonne National Laboratory, 9700 South Cass Avenue, Argonne, IL 60439

58. Ake Bjorck, Department of Mathematics, Linkoping University, S-581 83 Linkoping, Sweden

59-63. Jean R. S Blair, Department of Computer Science, Ayres Hall, University of Tennessee, Knoxville, TN 37996-1301

64. Heather Booth, Department of Computer Science, Ayres Hall, University of Ten. nessee, Knoxville, 'TN 37996-1301

65. Roger W. Brockett, Wang Professor of Electrical Engineering and Computer Science, Division of Applied Sciences, Harvard University, Cambridge, MA 02138

66. James C. Browne, Department of Computer Science, University of Texas, Austin, TX 78712

67. Bill L. Buzbee, Scientific Computing Division, National Center for Atmospheric Research, P.O. Box 3000, Boulder, CO 80307

68. Donald A. Calahan, Department of Electrical and Computer Engineering, University of Michigan, Ann Arbor, MI 48109

69. John Cavallini, Acting Director, Scientific Computing Staff, Applied Mathematical Sciences, Office of Energy Research, U.S. Department of Energy, Washington, DC 20585

70. Ian Cavers, Department of Computer Science, University of British Columbia, Vancuuver, British Columbia V6T 1W5, Canada

71. 'Tony Chan, Department of Mathematics, University of California, Los Angeles, 405 Hilgard Avenue, Los Angeles, CA 90024

72. Jagdish Chandra, Army Research Office, P.O. Box 12211, Research Triangle Park, NC 27709

73. Eleanor Chu, Department of Computer Science, University of Waterloo, Waterloo, Ontario, Canada N2L 3G1

74. Melvyn Ciment, National Science Foundation, 1800 G Street N.W., Washington, DC 20550

75. Tom Coleman, Department of Computer Science, Cornell University, Ithaca, NY 14853

76. Paul Concus, Mathematics and Computing, Lawrence Berkeley Laboratory, Berkeley, CA 94720

77. Andy Conn, IBM 'I. J. Watson Research Center, P.O. Box 218, Yorktown Heights, NY 10598

78. John M. Conroy, Supercomputer Research Center, 17100 Science Drive, Bowie, MD 20715-4300

79. Jane K. Cullum, IBM T. J. Watson Research Center, P.O. Box 218, Yorktown Heights, NY 10598 
80. George Cybenko, Department of Math and Computer Science, Dartmouth College, Hanover, NH 03755

81. George J. Davis, Department of Mathematics, Georgia State University, Atlanta, GA 30303

82. Tim A. Davis, Computer and Information Sciences Department, 301 CSE, University of Florida, Gainesville, Florida 32611.2024

83. John J. Dorning, Department of Nuclear Engineering Physics, Thornton Hall, McCormick Road, University of Virginia, Charlottesville, VA 22901

84. Iain Duff, Numerical Analysis Group, Central Computing Department, Atlas Centre, Rutherford Appleton Laboratory, Didcot, Oxon OX 11 0QX, England

85. Patricia Eberlein, Department of Computer Science, SUNY at Buffalo, Buffalo, NY 14260

86. Stanley Eisenstat, Department of Computer Science, Yale University, P.O. Box 2158 Yale Station, New Haven, CT 06520

87. Lars Elden, Department of Mathematics, Linkoping University, 58183 Linkoping, Sweden

88. Howard C. Elman, Ccmputer Science Department, University of Maryland, College Park, MD 20742

89. Robert E. England, Mathematics and Computer Science Department, Northern Kentucky University, Highland Heights, KY 41076-1448

90. Albert M. Erisman, Boeing Computer Services, P.O. Box 24346, M/S 7L-21, Seattle, WA 98124-0346

91. Ia; Foster, Mathematics and Computer Science Division, Argonne National Labcratory, 9700 South Cass Avenue, Argonne, IL 60439

92. Geoffrey C. Fox, Northeast Parallel Architectures Center, 111 College Place, Syracuse University, Syracuse, NY 13244-4100

93. Paul O. Frederickson, Center for Research on Parallel Computation, MS B:?87, Los Alamos National Laboratory, Los Alamos, NM 87545

94. Fred N. Fritsch, Computing \& Mathematics and Statistics Division, Lawrence Livermore National Laboratory, P.O. Box 808, L-316 Livermore, CA 94550

95. Robert E. Funderlic, Department of Computer Science, North Carolina State University, Raleigh, NC 27650

96. K. Gallivan, Computer Science Department, University of Illinois, Urbana, IL 61801

97. Dennis B. Gannon, Computer Science Department, Indiana University, Bloomington, IN 47405

98. Feng Gao, Department of Computer Science, University of British Columbia, Vancouver, British Columbia V6T 1W5, Canada

99. David M. Gay, Bell Laboratories, 600 Mountain Avenue, Murray Hill, NJ 07974 
100. C. William Gear, Computer Science Department, University of Illinois, Urbana, IL 61801

101. W. Morven Gentleman, Division of Electrical Engineering, National Research Council, Building M-50, Room 344, Montreal Road, Ottawa, Oritario, Canada K1A $0 R 8$

102. J. Alan George, Vice President, Acadernic and Provost, Needles Hall, University of Waterloo, Waterloo, Ontario, Canada N2L 3G1

103. John R. Gilbert, Xerox Palo Alto Research Center, 3333 Coyote Hill Road, Palo Alto CA 94304

104. Gene H. Golub, Department of Computer Science, Stanford University, Stanford, CA 94305

105. Joseph F. Grcar, Division 8245, Sandia National Laboratories, Livermore, CA 94551-0966

106. John Gustafson, 236 Wilhelm, Ames Laboratory, lowa State University, Ames, IA 50011

107. Per Christian Hansen, UCI*C Lyng,by, Building 305, Technical University of Denmark, DK-2800 Lyngby, Denmark

108. Richard Hanson, IMSL Inc., 2500 Park West Tower One, 2500 City West Blyd., Houston, 'TX 77042-3020

109. Michael T. Heath, NCSA, University of Illinois, 4157 Beckman Institute, 405 North Matthews Avenue, Urbana, IL 61801-2300

110. Don E. Heller, Physics and Computer Science Department, Shell Development Co., P.O. Box 481, Houston, TX 77001

111. Nicholas J. Higham, Department of Mathematics, University of Manchester, Grt Manchester, M13 9FL, England

112. Charles J. Holland, Air Force Office of Scientific Research, Building 410, Bolling Air Force Base, Washington, DC 20332

113. Robert E. Huddleston, Computation Department, Lawrence Livermore National Laboratory, P.O. Box 808, Livermore, CA 94550

114. John Huseby, Cray Research Superservers, Inc., 3601 S. W. Murray Blvd., Beaver. ton, OR 97005

115. Ilse Ipsen, Department of Computer Science, Yale University, P.O. Box 2158 Yale Station, New Haven, CT' 06520

116. Elizabeth Jessup, University of Colorado, Department of Computer Science, Boulder, CO 80309-0430

117. Barry Joe, Department of Computer Science, University of Alberta, Edmonton, Alberta T6G 2ll1, Canada

118. Lennart Johnsson, Thinking Machines Inc., 245 First Street, Cambridge, MA $02142-1214$ 
119. Harry Lordan, Department of Electrical and Computer Engineering, University of Colorado, Boulder, CO 80509

120. Bo Krgstrom, Institute of Information Processing, University of Umea, 5-901 87 Umea, Sweden

121. Malvyn H. Kalos, Cornell Theory Center, Engineering and Theory Center Bldg,, Cornell University, Ithaca, NY 14853-3901

122. Hans Kaper, Mathematics and Computer Science Division, Argonne National Laboratory, 9700 South Cass A venue, Bldg. 221, Argonne, IL 60439

123. Linda Kaufman, Bell Laboratories, 600 Mountain Avenue, Murray Hill, NJ 07974

124. Robert J. Kee, Division 8245, Sandia National Laboratories, Livermore, CA 945510969

125. Kenneth Kennedy, Department of Computer Science, Rice University, P.O. Box 1892, Houston, TX 77001

126. Eric S. Kirsch, Department of Computer Science, Ayres Hall, University of Tennessee, Knoxville, TN 37996-1301

127. Thomas Kitchens, Department of Energy, Scientific Computing Staff, Office of Energy Research, ER-7, Office G-236 Germantown, Washington, DC 20585

128. Michael A. Langston, Department of Computer Science, Ayres Hall, University of Tennessee, Knoxville, TN 37996-1301

129. Richard Lau, Office of Naval Research, Code 1111MA, 800 N. Quincy Street, Boston Tower 1 Arlington, VA 22217-5000

130. Alan J. Laub, Department of Electra : and Computer Engineering, University of California, Santa Barbara, CA 93106

131. Robert L. Launer, Army Research Office, P.O. Box 12211, Research Triangle Park, NC 27709

132. Charles Lawson, MS 301-490, Jet Propulsion Laboratory, 4800 Oak Grove Drive, Pasadena, CA 91109

133. Peter D. Lax, Courant Institute of Mathematical Sciences, New York University, 251 Mercer Street, New York, NY 10012

134. James E. Leiss, Rt. 2, Box 142C, Broadway, VA 22815

135. John G. Lewis, Boeing Computer Services, P.O. Box 24346, M/S 7L-21, Seattle, WA $98124-0346$

136. Jing Li, IMSL Inc., 2500 Park West Tower One, 2500 City West Blvd., Houston, TX 77042-3020

137. Heather M. Liddell, Center for Parallel Computing, Department of Computer Science and Statistics, Queen Mary College, University of London, Mile End Road, London E1 4NS, England

138. Arno Liegmann, c/o ETH Rechenzentrum, Clausiusstr. 55, CH-8092 Zurich, Swizzerland 
139. Joseph Liu, Department of Computer Science, York University, 4700 Keele Street, North York, Ontario, Canada M3J 1P?

140. Robert F. Lucas, Supercomputer Research Center, 17100 Science Drive, Bowie, MD 20715-4300

141. Franklin Luk, Electrical Engineering Department, Cornell University, Ithaca, NY 14853

142. Brian A. Malloy, 216 Duke Street, Clemson, SC 29631

143. Thomas A. Manteuffel, Department of Mathematics, University of Colorado Denver, Campus Box 170, P.O. Box 173364, Denver, CO 80217-3364

144. James McGraw, Lawrence Livermore National Laboratory, L-306, P.O. Box 808, Livermore, CA 94550

145. Paul C. Messina, Mail Code 158-79, California Institute of Technology, 1201 E. California Blvd., Pasadena, CA 91125

146. Cleye Moler, The Mathworks, 24 Prime Park Way, Natick, MA 0176

147. Neville Moray, Department of Mechanical and Industrial Engineering, University of Illinois, 1206 West Green Street, Urbana, IL 61801

148. Dianne P. O'Leary, Computer Science Department, University of Maryland, College Park, MD 20742

149. James M. Ortega, Department of Applied Mathernatics, Thornton Hall, University of Virginia, Charlottesville, VA 22901

150. Charles F. Osgood, National Security Agency, Ft. George G. Meade, MD 20755

151. Steve Otto, Department of Computer Sci. \& Eng., Oregon Graduate Institute, 19600 N.W. von Neumann Drive, Beaverton, OR 97006-1999

152. Chris Paige, OADDR, McCill University, McConnell Engineering Building 3480 University Street Montreal, PQ Canada H3A 2A7

153. Roy P. Pargas, Department of Computer Science, Clemson University, Clemson, SC 29634-1906

154. Beresford N. Parlett, Department of Mathematics, University of California, Berkeley, CA 94720

155. Merrell Patrick, Department of Computer Science, Duke University, Durham, NC 27706

156. Daniel J. Pierce, Boeing Computer Services, P.O. Box 24346, M/S 7L-21, Seattle, WA 98124-0346

157. Robert J. Pemmons, Departments of Mathematics and Computer Science, Box 7311, Wake Forest University Winston-Salem, NC: 27109

158. Jesse Poore, Department of Computer Science, Ayres Hall, University of I'nnessee, Knoxville, TN 37996-1301

159. Alex Pothen, Department of Computer Science, Pennsylvania State University, University Park, PA 16802 
160. Yuanchang Qi, IBM European Petroleum Application Center, P.O. Box 585, N4040 Hafrsfjord, Norway

161. Giuseppe Radicati, IBM European Center for Scientific and Engineering Computing, via del Giorgione 159, J-00147 Roma, Italy

162. S. S. Ravi, Department of Computer Science, Ll67A, 1400 Washington Avenue, Albany, NY 12222

163. John K. Reid, Numerical Analysis Group, Central Computing Department, Atlas Centre, Rutherford Appleton Laborato., Didcot, Oxon OX11 0QX, England

164. Werner C. Rheinboldt, Department of Mathematics and Statistics, University of Pittsburgh, Pittsburgh, PA 15260

165. John R. Rice, Computer Science Department, Purdue University, West Lafayette, IN 47907

160. Garry Rodrigue, Numerical Mathematics Group, Lawrence Livermore Laboratory, Livermore, CA 94550

167. Donald d. Rose, Department of Computer Science, Duke University, Durharn, NC 27706

168-172. Bill Rosener, Department of Computer Science, Ayres Hall, University of Tennessee, Knoxville, TN 37996-1301

173. Idward Rothberg, Department of Computer Science, Stanford University, Stanford, CA 94305

174. Axel Ruhe, Department of Computer Science, Chalmers University of Technology, S-41206 Goteborg, Sweden

175. Joel Saltz. ICASE, MS 132C, NASA Langley Research Center, Hampton, VA. 23605

176. Ahmed H. Sameh, Cenler for Supercomputing R\&D, 1384 W. Springfield Avenue, University of Illinois, Urbana, IL 61801

177. Michael Saunders, Systems Optimization Laboratory, Operations Research Department, Stanford University, Stanford, CA 94305

178. Robert Schreiber, RIACS, Mail Stop 230-5, NASA Ames Research Center, Moffet Field, CA 94035

179. Martin 11. Sctultz, Department of Computer Science, Yale University, P.O. Box. 2158 Yale Station, New Haven, CT 06520

180. David S. Scott. Intel Scientific Computers, 15201 N.W. Greenbrier Parkway, Beaverton, OR. 97006

181. Lawrence F. Shampine, Mathematics Department, Southern Methodist University, Dallas, TX 75278

182. Andy Sherman. Department of Computer Science, Yale University, P.O. Box 2158 Yale Station. New Haven, Cr 06520 
183. Kermit Sigmon, Department of Mathematics, University of Florida, Gainesville, FL 32611

184. Horst Sirnon, Mail Stop T045-1, NASA Ames Research Center, Moffett Field, CA 94035

185. Anthony Skjellum, Lawrence Livermore National Laboratory, 7000 East Ave., L316, P.O. Box 808 Livermore, CA 94551

186. Danny C. Sorensen, Department of Mathematical Sciences, Rice University, P. O. Box 1892, Houston, TX 77251

187. G. W. Stewart, Computer Science Department, University of Maryland, College Park, MD 20742

188. Paul N. Swartztrauber, National Center for Atmospheric Research, P.O. Box 3000, Boulder, $\mathrm{CO} 80307$

189. Michael G. Thomason, Department of Computer Science, Ayres Hall, University of 'Tennessee, Knoxville, TN 37996-1301

190. Philippe Toint, Lepartment of Mathematics, University of Namur, FUNOP, 61 rue de Bruxelles, B-Namur, Belgium

191. Bernard Tourancheau, LIP, ENS-Lyon, 69364 Lyon cedex 07, France

192. Hank Van der Vorst, Department of Techn. Mathematics and Computer Science, Delft University of 'Technology, P.O. Box 356, NL-2600 AJ Delft, The Netherlands

193. Charles Van Loan, Department of Computer Science, Cornell University, Ithaca, NY 14853

194. Jim M. Varah, Centre ior Integrated Computer Systems Research, University of British Columbia, Office 2053-2324 Main Mall, Vancouver, British Columbia V6T IW5, Canada

195. Udaya B. Ven.'lapati, Department of Computer Science, University of Central Florida, Orlando, FL 32816-0362

196. Robert G. Voigt, National Science Foundation, Room 417, 1800 G Street, N.W., Washington, DC 20550

197. Phuong Vu, Gray Research, Iur., 1345 Northland Dr., Mendota Heights, MN 55120

198. Daniel D. Warner, Department of Mathematical Sciences, O-104 Martin Hall, Clemson University, Clemson, Sc: 29631

199. Gilber: G. Weigand, Computing Systems Technology Office, Defense Advanced Research Projects Agency, 3701 North Fairfax Drive, Arlington, VA 22203-1714

200. Mary F. Wheeler, Rice University, Department of Mathematical Sciences, P.O. Box 1892, Houston, TX 77251

201. Andrew B. White, Computing Division, Los Alamos National Laboratory, P.O. Box 1663, MS-265, Los Alamos, NM 87545 
202. Michael Wolfe, Department of Computer Sci. \& Eng., Oregon Graduate Institute, 19600 N.W. von Neumann Drive, Beaverton, OR 97006-1999

203. Margaret Wright, Bell Laboratories, 600 Mountain Avenue, Murray Hill, NJ 07974

204. David Young, University of Texas, Center for Numerical Analysis, RLM 13.150, Austin, TX 78731

205. Earl Zmijewski, Department of Computer Science, University of California, Santa Barbara, CA 93106

206. Office of Assistant Manager for Energy Research and Development, U.S. Department of Energy, Oak Ridge Operations Office, P.O. Box 2001 Oak Ridge, TN 37831-8600

207-216. Office of Scientific \& Technical Information, P.O. Box 62, Oak Ridge, TN 37831 

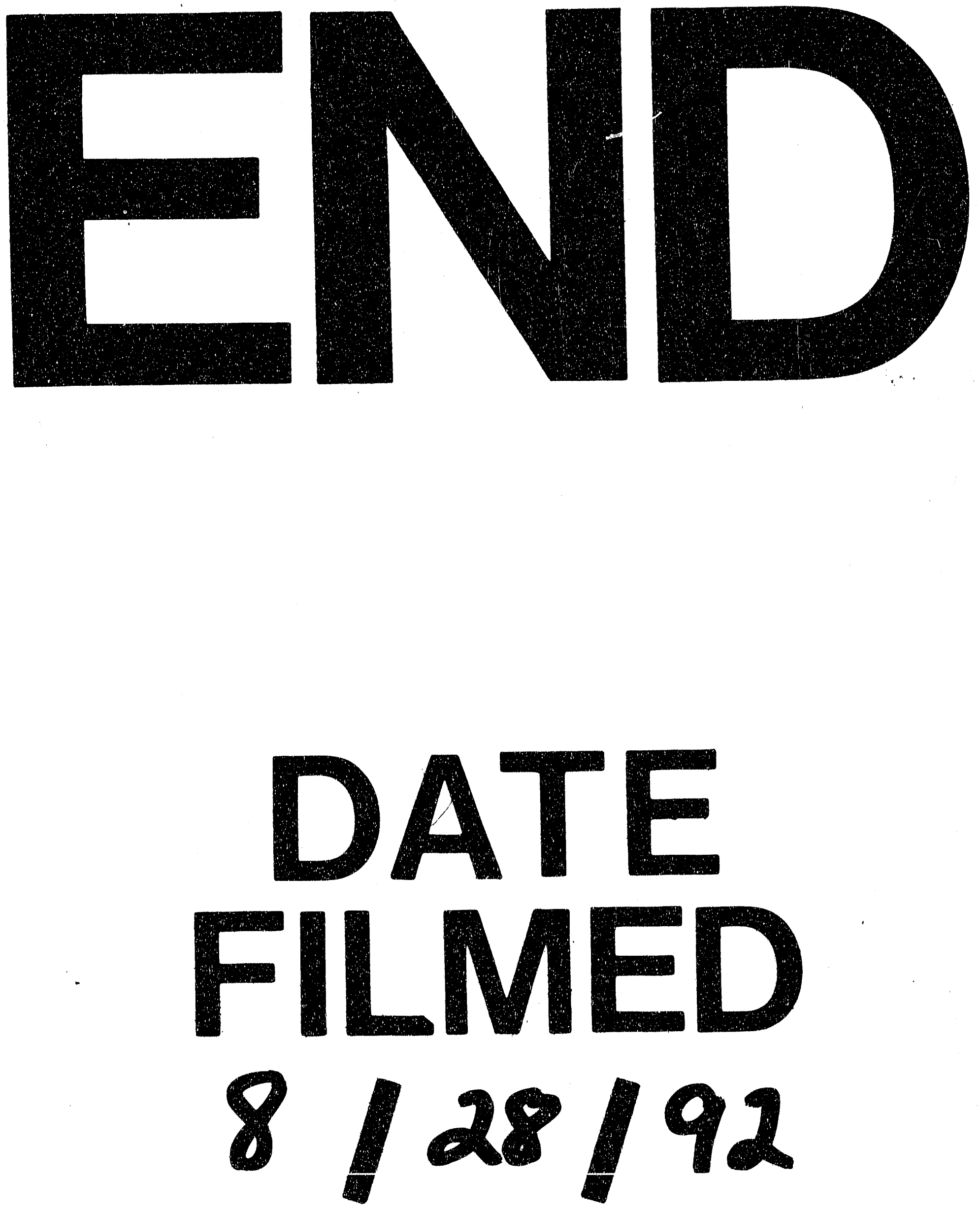
